Since for any positive integer $m$,

$$
\text { LV (1990) }
$$

$$
L(2 m+1, \bar{\chi})=\frac{(-1)^{m} i \tau(\bar{\chi})}{2(2 m+1) !}\left(\frac{2 \pi}{q}\right)^{2 m+1} B_{2 m+1, \chi}, \quad|L(2 m+1, \bar{\chi})|<\zeta(3),
$$

we find that

$$
|S|<C_{2}(n) q^{n+1 / 2}
$$

in the same way as above. Then our assertion follows immediately from (5), (8) and (9).

3. Proof of Theorem 3. In the cases $n=1$ and $n=2$, our assertion follows from (3), (6), (7) and the result of Pintz [2], because $S_{\chi}(1)=q B_{1, \chi}$ and $S_{\chi}(2)=q S_{\chi}(1)$. [2].

If $n \geqslant 3$, our assertion follows easily from Theorem 2 and the result of Pintz

\title{
References
}

[1] K. Iwasawa, Lectures on p-adic L-functions, Ann. of Math. Stud. 74, Princeton Univ. Press, 1972.

[2] J. Pintz, Elementary methods in the theory of L-functions VI, Acta Arith. 32(1977), 397-406.

[3] K. S. Williams, A class of character sums, J. London Math. Soc. 46 (1971), 67-72.

DEPARTMENT OF MATHEMATICS, TOYO UNIVERSITY

Kawagoe-Shi, Saitama 350, Japan

\section{Indépendance linéaire \\ des valeurs des solutions transcendantes de certaines équations fonctionnelles II}

par

\section{Jean-Paul Bézivin (Paris)}

0. Introduction. Dans cet article, nous poursuivons l'étude commencée dans [2] des propriétés arithmétiques de certaines fonctions entières transcendantes.

Bien que tout ce que nous allons montrer soit valable pour un corps quadratique imaginaire, nous nous bornerons à considérer le cas du corps $\mathbf{Q}$ des nombres rationnels, la généralisation à un corps quadratique imaginaire étant immédiate. Nous notons $\overline{\boldsymbol{Q}}$ une clôture algébrique de $\boldsymbol{Q}$.

Soit $u(n)$ une suite récurrente linéaire d'éléments de $\boldsymbol{Q}$, c'est-à-dire une suite d'éléments de $\boldsymbol{Q}$ ayant une expression de la forme

$$
u(n)=\sum_{i=1}^{s} P_{i}(n) a_{i}^{n}
$$

avec $P_{i}$ appartenant à $\bar{Q}[x]$, non nuls, et les $a_{i}$ à $\bar{Q}-\{0\}$. Nous supposerons dans tout cet article que $u(n)$ est non nul pour tout entier $n$.

Nous notons $A(n)$ la suite définie par

$$
A(n)=u(0) \ldots u(n) .
$$

Les fonctions qui nous intéressent sont les fonctions de la forme

$$
f(z)=\sum_{n=0}^{\infty} z^{n} / A(n) .
$$

Nous supposerons aussi dans toute la suite que la suite $u(n)$ est non dégénérée, c'est-à-dire qu'aucun des $a_{i}$ n'est une racine de l'unité différente de 1 , et de même pour les quotients $a_{i} / a_{j}$.

Sous ces hypothèses, la fonction $f(z)$ est une fonction entière de la variable Complexe $z$, et nous nous intéressons aux propriétés d'indépendance linéaire sur $\boldsymbol{Q}$ de $f$ et de ses dérivées aux points de $\boldsymbol{Q}$.

Les fonctions $f(z)$ satisfont à certaines équations fonctionnelles, voir [2],

d'où le titre. L'étude faite dans [2] concernait le cas où les polynômes $P_{i}$ figurant dans l'expression (1) étaient constants. 
Nous ne ferons plus une telle hypothèse dans cet article, mais nous serons contraints de conserver une hypothèse de fréquence dominante portant sur les $a_{i}$ (voir plus loin).

D'ailleurs, un des ingrédients de la preuve du résultat d'indépendance linéaire sera une version approchée du Théorème du Quotient de Hadamard, que nous établirons sous cette hypothèse en utilisant une technique due $\grave{a}$ C. Pisot (voir [1]).

Nous renvoyons à [2] pour des références bibliographiques sur des résultats semblables à ceux démontrés dans cet article.

I. Notations et énoncés des résultats. Dans cette partie nous donnons les notations et les énoncés des résultats. Soit $u(n)$ la suite récurrente linéaire définie par l'expression (1). Nous ferons dans toute la suite les hypothèses suivantes:

(a) $u(n)$ est à valeurs dans $\boldsymbol{Q}$.

(b) Les éléments $a_{i}$ sont entiers algébriques.

(c) $u(n)$ est non dégénérée.

(d) On a $\left|a_{1}\right|>\left|a_{i}\right| \geqslant 1, i=2, \ldots, s$, et si $\left|a_{i}\right|=1$, alors $a_{i}=1$.

Les nombres algébriques $a_{i}$ sont les fréquences de la suite récurrente $u(n)$; l'hypothèse (d) est donc l'hypothèse de la fréquence dominante.

Le résultat principal de l'article est le suivant:

THÉORÈME 1. Soient $t$ et $m$ deux entiers naturels, avec $t$ non nul, et $b_{1}, \ldots, b_{1}$ des éléments distincts de $Q-\{0\}$. Soit $G$ le sous-groupe multiplicatif de $\bar{Q}$ engendré par $a_{1}, \ldots, a_{s}$. On suppose que, pour i différent de $j$, le quotient $b_{i} / b_{j}$ n'appartient pas à $G$. On fait enfin l'hypothèse que si la suite $u(n)$ a la fréquence 1 , que l'on peut supposer être $a_{s}$, et si $P_{s}$ est un polynôme constant, alors $P_{s}$ n'est pas de la forme $\zeta b_{k}$ avec $\zeta$ appartenant à $G$. Alors la famille $\left\{1, f^{(j)}\left(b_{i}\right), 0 \leqslant j \leqslant m\right.$. $1 \leqslant i \leqslant t\}$ est $Q$-linéairement indépendante.

Nous avons déjà parlé du théorème du quotient de Hadamard; nous allons maintenant donner son énoncé. Il s'agit d'un résultat qui avait été conjecturé par C. Pisot, et qui -a été finalement démontré par Y. Pourchet et A. J. van der Poorten ([5], [7], [8]). Voir aussi la rédaction complète qui en a été donnée par R. Rumely dans [6].

THÉORÈme DU QUOTIENT DE HADAMARD. Soit $K$ un corps de caractéristique nulle et $u(n), w(n)$ deux suites récurrentes linéaires à valeurs dans $K$. On suppose que $v(n)$ est non nulle pour toute valeur de n, et qu'il existe un sous-anneau E de $K$, de type fini sur $Z$, tel que le quotient $w(n) / v(n)$ appartienne à $E$ pour tout $n$. Alors la suite $w(n) / v(n)$ est récurrente linéaire.

La version approchée de ce résultat dont nous aurons besoin est la suivante:

THÉORÈme 2. Soient $w(n)$ et $v(n)$ deux suites récurrentes linéaires à valeurs dans $\boldsymbol{Q}$. On suppose que $v(n)$ est non nulle pour tout $n$, et que cette suite possède une fréquence dominante. On note Tle sous-groupe multiplicatif engendré par les fréquences de $v(n)$, et $S$ l'ensemble des fréquences de $w(n)$. On vérifie facilement que $U=T S$ est stable sous l'action du groupe de Galois de $\overline{\boldsymbol{Q}}$ sur $\boldsymbol{Q}$. On suppose de plus que l'on peut écrire $w(n) / v(n)=c(n)+d(n)$ avec $c(n)$ élément de $\mathbf{Z}$ pour lout $n$ et $d(n)$ une suite d'éléments de $\boldsymbol{Q}$ telle que $d(n)=O\left(\varrho^{n}\right)$ avec $0<\varrho<1$.

Alors la suite $c(n)$ est une suite récurrente linéaire, dont les fréquences appartiennent à $U=T S$.

La suite de l'article est organisée ainsi: Dans la partie II, nous donnons la démonstration du théorème 2 . La partie III est consacrée à la démonstration du théorème 1. Enfin la partie IV est dévolue à quelques remarques.

II. Preuve du théorème 2. Nous démontrons donc maintenant le théorème 2. Nous notons

$$
v(n)=P_{1}(n) \omega_{1}^{n}+\ldots+P_{h}(n) \omega_{h}^{n} .
$$

$0_{\text {n }}$ a donc d'après les hypothèses faites, $\left|\omega_{1}\right|>\left|\omega_{i}\right|$ pour $i$ différent de 1 , et $\left|\omega_{1}\right|>1$.

Soit $N$ un entier assez grand que nous préciserons plus loin. Si l'entier $n$ est assez grand, $P_{1}(n)$ est non nul, et la suite

$$
r(n)=-\left(P_{2}(n) \omega_{2}^{n}+\ldots+P_{h}(n) \omega_{h}^{n}\right) /\left(P_{1}(n) \omega_{1}^{n}\right)
$$

est de module strictement inférieur à 1 . On peut alors écrire

$$
P_{1}(n)^{N} w(n) / v(n)=\sum_{i=0}^{N} \omega_{1}^{-n} P_{1}(n)^{N} w(n) r(n)^{i}+P_{1}(n)^{N} r(n)^{N+1} w(n) / v(n) .
$$

Chacune des suites $\omega_{1}^{-n} P_{1}(n)^{N} w(n) r(n)^{i}$ est une suite récurrente linéaire; pour tout réel positif $c$, il existe un entier $N$ tel que la suite $P_{1}(n)^{N} r(n)^{N+1} w(n) / v(n)$ Soit un $O\left(c^{n}\right)$ quand $n$ tend vers l'infini, en raison de l'hypothèse de frequence ominante. Il en résulte que l'on peut écrire, pour un entier $N$ convenable,

$$
P_{1}(n)^{N} c(n)=H_{N}(n)+J_{N}(n)
$$

Oủ la suite $H_{N}(n)$ est une suite récurrente linéaire d'éléments de $\bar{Q}$, à fréquences dans $U=S T$, et où la suite $J_{N}(n)$ est un $O\left(c_{1}^{n}\right)$, avec $0<c_{1}<1$.

Le polynôme $P_{1}$ a ses coefficients dans $\bar{Q}$. Quitte à multiplier encore par un polynôme, on peut supposer que l'on a (4)

$$
Q(n) c(n)=H(n)+J(n),
$$

avec $Q(n)$ polynôme à coefficients dans $Z$, non nul, $H(n)$ une suite récurrente linéaire d'éléments de $\bar{Q}$, et $J(n)$ une suite qui est un $O\left(c_{2}^{n}\right)$ avec $0<c_{2}<1$.

Du fait que $H(n)$ est une suite récurrente linéaire à valeurs dans $\bar{Q}$, il existe un entier $e$ dans $N$ et des entiers rationnels non tous nuls $m_{i}$ tels que l'on ait $\sum_{i=0}^{e} m_{i} H(n+i)=0$. On peut choisir la longueur $e$ de cette récurrence de façon 
que les zéros du polynôme $\sum m_{i} x^{i}$ soient des conjugués de fréquences de $H^{(n)}$ donc dans $U=T S$

De la relation (4) on tire alors

$$
\sum_{i=0}^{e} m_{i} Q(n+i) c(n+i)=\sum_{i=0}^{e} m_{i} J(n+i) .
$$

Dans (5), l'expression de gauche est un élément de $Z$. Le membre de droite tend vers zéro si $n$ tend vers l'infini. Il en résulte que les deux membres de l'expression (5) sont nuls pour $n$ assez grand, et ceci prouve que la suite $Q(n) c^{(n)}$ est une suite récurrente linéaire.

Nous pouvons maintenant appliquer le Théorème du quotient de Hadamard: la suite $c(n)$ est à valeurs dans $Z$ pour tout $n$, et est le quotient de la suite récurrente linéaire $Q(n) c(n)$ par la suite récurrente linéaire $Q(n)$, et par suite $e^{5 t}$ elle-même récurrente linéaire.

D'autre part, les fréquences de $c(n)$ seront alors des fréquences de $Q(n) c(n)$ donc des racines de $\sum m_{i} x^{i}$ et par suite dans $U=T S$, ce qui termine démonstration du théorème 2 .

III. Démonstration du théorème 1. Nous aurons besoin du résultat suivant:

Lemme 1. Pour $R$ réel positif on pose $|f|(R)=\operatorname{Max}\{|f(z)|,|z| \leqslant R\}$. On $a$ alors l'estimation

$\log |f|(R) \leqslant(\log R)^{2} /\left(2 \log \left|a_{1}\right|\right)-q \log R \log \log R / \log \left|a_{1}\right|+O(\log R)$,

où $q$ est le degré du polynôme $P_{1}$ coefficient de $a_{1}^{n}$ dans (1).

Démonstration. De l'hypothèse de la fréquence dominante on tire facilement qu'il existe une constante $c_{3}$ positive telle que l'on ait pour tout entier $n$ la minoration

$$
|u(n)| \geqslant c_{3} n^{q}\left|a_{1}\right|^{n} .
$$

Par suite on a pour $A(n)$ la minoration

$$
|A(n)| \geqslant c_{4} c_{3}^{n}(n !)^{q}\left|a_{1}\right|^{n(n+1) / 2} .
$$

On a donc pour $z$ de module inférieur ou égal à $R$, l'inégalité

$$
|f(z)| \leqslant \sum_{n \geqslant 0} c_{4}^{-1} c_{3}^{-n}(n !)^{-q}\left|a_{1}\right|^{-n(n+1) / 2} R^{n} .
$$

Il suffit alors de démontrer l'inégalité du lemme 1 pour la fonction $U(R)$ égale

$$
U(R)=\sum_{n \geqslant 1}(n !)^{-q}\left|a_{1}\right|^{-n(n+1) / 2} R^{n} .
$$

Il existe une constante positive $c_{5}$ telle que l'on ait

$$
U(R) \leqslant \sum_{n \geqslant 1} \exp \left(n \log R-q n \log n-n^{2} \log \left|a_{1}\right| / 2+c_{5} n\right) .
$$

Soit $\beta(x)$ la fonction de la variable réelle positive

$$
\beta(x)=x \log R-q x \log x-x^{2} \log \left|a_{1}\right| / 2+c_{5} x .
$$

le maximum de cette fonction est atteint au point $x(R)$ qui vérifie l'égalité

$$
x \log \left|a_{1}\right|+q \log x=\log R+c_{5}-q .
$$

II est facile de voir que l'on peut écrire

$$
x(R)=(\log R-q \log \log R) / \log \left|a_{1}\right|+\tau(R),
$$

${ }^{0} \mathrm{u} \tau$ est une fonction bornée quand $R$ tend vers l'infini. Il en résulte que chacun

des termes du membre de droite de l'inégalité (8) est majoré par une expression

de la forme

$$
\exp \left((\log R)^{2} / 2 \log \left|a_{1}\right|-q \log R \log \log R / \log \left|a_{1}\right|+c_{6} \log R\right),
$$

${ }^{0} \mathrm{U}_{6} c_{6}$ est une constante positive.

Soit maintenant $y(R)$ la partie entière de $3 \log R / \log \left|a_{1}\right|$. Il est immédiat que, pourvu que $R$ soit assez grand, la fonction $\beta(x) / x$ est plus petite que -1 Pour $x$ plus grand que $y(R)$. Il en résulte que le membre de droite de l'inégalité

(8) est majoré par l'expression

$$
y(R) \exp \left((\log R)^{2} / 2 \log \left|a_{1}\right|-q \log \log R / \log \left|a_{1}\right|+c_{6} \log R\right)+c_{7},
$$

${ }^{0} i_{c} c_{7}$ est une constante indépendante de $R$, pourvu que $R$ soit assez grand. On

en déduit facilement l'inégalité du lemme 1.

Nous passons maintenant à la preuve du théorème 1 .

Nous allons raisonner par l'absurde, en supposant donc que la famille $\left\{1, f^{(i)}\left(b_{j}\right)\right\}$ est $Q$-linéairement dépendante. Il est facile de voir que ceci est quivalent à dire que la famille $\left\{1, \tau^{i}(f)\left(b_{j}\right)\right\}$ est $Q$-linéairement dépendante (on rappelle que $\tau$ est l'opérateur différentiel $x d / d x$ ). Il existe donc des éléments $e$ et

$e_{i, j}$ dans $\boldsymbol{Q}$, non tous nuls, tels que l'on ait

$e+\sum e_{i, j} \tau^{i}(f)\left(b_{j}\right)=0$

us définissons la fonction $g(z)$ par l'égalité

(10)

$$
g(z)=e+\sum e_{i, j} \tau^{i}(f)\left(b_{j} z\right) .
$$

$L_{a}$ fonction $g(z)$ est une fonction entière de la variable complexe $z$, qui vérifie $\theta(1)=0$; par suite la fonction $h(z)=g(z) /(z-1)$ est encore une fonction analytique entière.

Nous écrivons les développements de Taylor de $g$ et $h$ sous la forme

$$
g(z)=\sum v(n) z^{n} / A(n), \quad h(z)=\sum w(n) z^{n} / A(n) .
$$

"l est facile de voir que pour $n$ entier naturel non nul, on a $v(n)=\sum e_{i, j} n^{i} b_{j}^{n}$, de sorte que $v(n)$ est une suite récurrente linéaire dont les fréquences sont parmi les $b_{k}$. 
Soient maintenant $\tilde{g}$ et $\tilde{h}$ les séries formelles définies par $\tilde{g}(x)=\sum v(n) x^{n}$ el $\tilde{h}(x)=\sum w(n) x^{n}$. La série $\tilde{g}$ est une fraction rationnelle; nous allons montref que la série $\tilde{h}$ a un rayon de convergence non nul dans $\boldsymbol{C}$.

Il existe deux constantes $\alpha$ et $\beta$, positives, telles que l'on ait pour toul $n$ entier l'inégalité $|u(n)| \leqslant \alpha \beta^{n}$. On en déduit que $|g|(R) \leqslant \alpha|f|(\beta R)$, et par suite que la fonction $|g|(R)$ vérifie une majoration semblable à celle du lemme

Il en est alors de même de la fonction $h$. D'après les inégalités de Cauchy, on a donc

\section{(11) $|w(n)| \leqslant|A(n)| \exp \left((\log R)^{2} / 2 \log \left|a_{1}\right|\right.$}

$$
\left.-q \log R \log \log R / \log \left|a_{1}\right|+c_{8} \log R-n \log R\right) \text {. }
$$

Dans cette inégalité, on prend $\log R=n \log \left|a_{1}\right|$, et après quelques calculs of trouve $|w(n)| \leqslant \exp \left(c_{9} n\right)$ pour $n$ assez grand, ce qui démontre l'assertion.

Nous traduisons maintenant l'égalité $g(z)=(z-1) h(z)$ sur les coefficients $w(n)$ et $v(n)$.

On trouve sans difficultés que $w(0)=-v(0)$ et que pour $n \geqslant 1$

$$
u(n) w(n-1)-w(n)=v(n) .
$$

Les deux suites $v(n)$ et $w(n)$ sont à valeurs dans $\boldsymbol{Q}$; les fréquences de la suite $u^{(n)}$ sont des entiers algébriques. Comme $v(n)$ est une suite récurrente linéaire, ${ }^{\text {of }}$ peut trouver un entier naturel $d$ non nul tel que $d^{n+1} v(n)$ et $d u(n)$ soient dans $Z$ pour tout $n$; il en résulte facilement que $d^{n+1} w(n)$ est dans $Z$ pour tout ${ }^{n}$. Nous procédons maintenant en deux étapes:

Etape 1: Nous allons montrer que la suite $w(n)$ est récurrente linéairé d'après ce qui précède, on ne restreint pas la généralité en supposant que les trois suites $u(n), v(n)$ et $w(n)$ sont à valeurs dans $\boldsymbol{Z}$.

Soit $m$ un entier naturel tel que $\left|a_{1}\right|^{m}>\exp \left(c_{9}\right)=c_{10}\left(c_{9}\right.$ est tel $q^{\text {ue }}$ $|w(n)| \leqslant \exp \left(c_{9} n\right)$ pour $n$ assez grand). En itérant la relation (12) on trouve que

$$
u(n+1) \ldots u(n+m) w(n)=w(n+m)+t(n),
$$

où $t(n)$ est la suite récurrente linéaire

$$
t(n)=v(n+m)+u(n+m) v(n+m-1)+\ldots+u(n+2) \ldots u(n+m) v(n+1) .
$$

On pose de plus $s(n)=u(n+1) \ldots u(n+m)$. La suite $s(n)$ est récurrente linéaire. et a une fréquence dominante qui est $a_{1}^{m}$. On peut donc écrire

$$
t(n) / s(n)=w(n)+p(n) \text {. }
$$

La suite $w(n)$ est à valeurs dans $Z$, et la suite $p(n)=-w(n+m) / s(n)$ est unt $O\left(\left(c_{10} a_{1}^{-m}\right)^{n}\right)$ et $\left|c_{10} a_{1}^{-m}\right|<1$. Nous sommes donc dans les conditions d'application du théorème 2 , et par conséquent la suite $w(n)$ est une suit récurrente linéaire.

Etape 2: Nous montrons que les résultats précédents conduisent à un na $^{\mathrm{e}}$ contradiction.
Nous écrivons $w(n)=\sum_{i=1}^{\lambda} Q_{i}(n) \omega_{i}^{n}$; les $\omega_{i}$ sont donc les fréquences de $w(n)$.

D'après le théorème 2 , tout $\omega_{i}$ peut s'écrire comme le produit d'un élément $\zeta$ de

$G$ et d'une fréquence $b_{j}$ de la suite $v(n)$, autrement dit on a $\omega_{i}=\zeta_{i} b_{k_{i}}$ pour tout $i$, avec $\zeta_{i}$ dans $G$.

Nous notons $i_{1}$ un indice tel que $\zeta_{i_{1}}$ soit de module maximal, et $i_{2}$ un indice tel que $\zeta_{i_{2}}$ soit de module minimal. On a donc

$$
\left|\zeta_{i_{1}}\right| \geqslant\left|\zeta_{i_{2}}\right| \text {. }
$$

Le nombre $a_{1} \omega_{i_{1}}=a_{1} \zeta_{i_{1}} b_{\boldsymbol{k}_{i_{1}}}$ est une fréquence de l'expression de gauche de l'égalité (12), en raison de la maximalité de $\zeta_{i_{1}}$. Par suite, c'est l'un des $b_{k}$. D'après les hypothèses faites sur les $b_{k}$, il en résulte que $k$ est égal à $k_{i_{1}}$, et par suite $\left|\zeta_{i}\right|=\left|a_{1}\right|^{-1}<1$.

Nous considérons maintenant $\omega_{i_{2}}$. Si cet élément n'apparaît pas comme fréquence du membre de gauche de l'expression (12), c'est qu'il est de la forme $a_{j} \omega_{i}$ (autrement dit, qu'il apparaît aussi comme fréquence dans le terme $u(n) w(n-1))$.

On a donc $\zeta_{i_{2}} b_{k_{i_{2}}}=a_{j} \zeta_{i} b_{k_{i}}$, d'où on déduit $k_{i_{2}}=k_{i}$, et ensuite que $a_{j}=1$, donc $j=s$.

Il faut donc que l'on ait $P_{s}(n) Q_{i_{2}}(n-1){\omega_{i_{2}}}^{-1}=Q_{i_{2}}(n)$ pour tout entier $n$, d'où on déduit que $P_{s}$ et $Q_{i_{2}}$ sont des polynômes constants, et par suite que la constante $P_{s}$ est égale à $\omega_{i_{2}}=\zeta_{i_{2}} b_{k_{i_{2}}}$, ce qui est exclus par l'une des hypothèses du théorème 1 .

Puisque $\omega_{i}$ est une fréquence du premier membre de l'égalité (12), c'est donc l'un des $b_{k}$. On en déduit alors que $\zeta_{i_{2}}$ est égal à 1. Par suite, on a $\left|\zeta_{i_{2}}\right|=1>\left|\zeta_{i_{1}}\right|$, ce qui est contradictoire avec l'inégalité (15), et cette contradiction termine la démonstration du théorème 1 .

IV. Remarques. 1) Nous donnons tout d'abord un exemple montrant que l'hypothèse supplémentaire du théorème 1 dans le cas où la suite $u(n)$ a la fréquence 1 est nécessaire.

On prend $u(n)=2^{n}+3$, la fonction $f(z)$ du théorème satisfait alors à la relation $f(2 z)+3 f(z)=z f(z)+1$. On a donc $f(6)=1$, autrement dit en prenant $b_{1}=6$, on a 1 et $f\left(b_{1}\right) \quad Q$-linéairement dépendants. On a bien sûr $P_{h}=3=2^{-1} b_{1}$ et $1 / 2$ appartient à $G$.

2) Nous avons considéré, quand $s=1$ dans les hypothèses du théorème 1 , uniquement le cas où $\left|a_{1}\right|>1$; on peut se demander ce qui se passe dans le cas où la suite $u(n)$ est égale à un polynôme non constant. Ce cas a été examiné dans [4]; la méthode conduit à des résultats semblables, et fait intervenir des propriétés de certains opérateurs différentiels.

3) Nous examinons maintenant ce qui paraît être l'hypothèse la plus contraignante du théorème 1 , à savoir l'hypothèse de la fréquence dominante. Cette hypothèse intervient dans le théorème 2 , et le lemme 1 . Pour ce qui 
concerne le théorème 2 , il faut voir que le cas où l'on n'avait pas de fréquence LV (1990) dominante était le cas difficile du théorème du quotient de Hadamard. La méthode utilisée dans ce cas ne semble pas devoir s'appliquer ici, et par suite il est probable qu'un tel résultat sera assez compliqué à démontrer.

Pour ce qui concerne le lemme 1 , il semble qu'une généralisation nécessite des renseignements précis sur la croissance d'une suite récurrente linéaire. Des résultats sur ce sujet ont été annoncés par H. P. Schlickewei et A. J. van der Poorten ([9]), mais les démonstrations n'ont pas été publiées semble-t-il à la connaissance de l'auteur.

Comme nous l'a fait remarquer le professeur A. Schinzel le théorème 2 de l'article de J. H. Evertse On sums of $S$-units and linear recurrences, Compositio Math. 53 (1984), 225-244, fournit dans le cas général une minoration de la forme (6), ce qui fait que le lemme 1 est en fait vrai sans l'hypothèse de la fréquence dominante.

4) Enfin remarquer que le résultat du théorème 2 peut se traduire comme une propriété de certains opérateurs fonctionnels; dans un autre cadre, nous avons fait une étude semblable dans [3].

\section{Bibliographie}

[1] B. Benzaghou, Algèbres de Hadamard, Bull. Soc. Math. France 98 (1970), 209-252.

[2] J.-P. Bézivin, Indépendance linéaire des valeurs des solutions transcendantes de certaines équations fonctionnelles, Manuscripta Math. 61 (1988), 103-129.

[3] - Solutions rationnelles de certaines équations fonctionnelles, Aequationes Math. 36 (1988), 112-124.

[4] J.-P. Bézi vin and P. R obba, A new p-adic method for proving irrationality and transcendence results, Ann. of Math. 129 (1989), 151-160.

[5] Y. Pourchet, Solution du probleme arithmétique du quotient de Hadamard de deux fractions rationnelles, C. R. Acad. Sci. Paris 288 (1979), 1055-1057.

[6] R. Rumely, Notes on van der Poorten's proof of the Hadamard Quotient Theorem, Sém. de Théorie des Nombres, Paris 1986-87, Progr. Math. 75, Birkhäuser, 1988, 349-409.

[7] A. J. van der Poorten, Hadamard operations on rational functions, Groupe d'étude d'analyse ultramétrique, 1982-83, exposé nº.

[8] - Solution de la conjecture de Pisot sur le quotient de Hadamard de deux fractions rationnelles, C. R. Acad. Sci. Paris 306 (1988), 97-102.

[9] H. P. Schlickewei and A. J. van der Poorten, The growth condition for recurrent sequences, Macquarie Math. Reports, 82-0041, North Ryde, Australia, 1982.

UNIVERSITE PIERRE ET MARIE CURIE

MATHÉMATIQUES

4. Place Jussieu, 75230 Paris Codox 05, France

\section{On the $(3 N+1)$-conjecture*}

by

\section{J. W. SANDER (Hannover)}

1. Introduction. We define for all odd positive integers $b$

$$
C(b)=(3 b+1) / 2^{e(b)}
$$

Where $2^{e(b)}$ is the greatest power of 2 dividing $3 b+1$. Obviously, $C(b)$ is odd again, thus we are able to define recursively

$$
C^{0}(b)=b, \quad C^{k+1}(b)=C\left(C^{k}(b)\right) \text { for } k \geqslant 0 .
$$

The following conjecture has been made.

$(3 N+1)$-CONJeCture. For all $b \in N, 2 \Varangle b$, there is a $k \in N_{0}$ such that $C^{k}(b)=1$.

Several results concerning the conjecture are known, for references see [4],

[5] and [7]. The problem itself is still open.

An odd positive integer $b$ will be called descending if $C^{k}(b)=1$ for some $k \in N_{0}$. In the first part of this paper we show that the conjecture is true, if one can prove for a certain "arbitrarily thin" congruence class that it contains only descending integers. In the second part we improve a lower bound of the number of descending integers below a given $x$ by R. E. Crandall [2].

2. A sufficient condition. Let $b$ be an odd positive integer. If $b$ is descending, We define

$$
S(b)=\min \left\{k \in N_{0}: C^{k}(b)=1\right\},
$$

Otherwise $S(b)=\infty$.

Lemma 1. For $b \in N, 2 \nmid b$, we have $S(b)=S(4 b+1)$.

Proof.

$$
C(4 b+1)=\frac{3(4 b+1)+1}{2^{e(4 b+1)}}=\frac{3 b+1}{2^{e(b)}}=C(b) .
$$

- Theorem 2 was first presented by the author at the Journées Arithmétiques 1987, Ulm, West Germany. 